CU-TP-804

SNUTP 96-124

hep-th/9612183

June 19, 2022

\title{
The BPS Domain Wall Solutions in Self-Dual Chern-Simons-Higgs Systems
}

\author{
Hsien-chung Kad \\ Department of Physics, Tamkang University, Tamsui, Taiwan 25137, R.O.C. \\ Kimyeong Lef \\ Department of Physics, Columbia University, New York, NY 10027, USA \\ Taejin Lę \\ Department of Physics, Kangwon National University, Chuncheon 200-701, Korea
}

\begin{abstract}
We study domain wall solitons in the relativistic self-dual Chern-Simons Higgs systems by the dimensional reduction method to two dimensional spacetime. The Bogomolny bound on the energy is given by two conserved quantities in a similar way that the energy bound for BPS dyons is set in some Yang-Mills-Higgs systems in four dimensions. We find the explicit soliton configurations which saturate the energy bound and their nonrelativistic counter parts. We also discuss the underlying $N=2$ supersymmetry.
\end{abstract}

PACS number: 11.27.+d, 11.10.Kk, 11.30.Pb

\footnotetext{
${ }^{1}$ electronic mail: hckao@mail.tku.edu.tw

${ }^{2}$ electronic mail: klee@phys.columbia.edu

${ }^{3}$ electronic mail: taejin@cc.akngwon.ac.kr
} 


\section{Introduction}

In past years the Abelian self-dual Chern-Simons Higgs system with an appropriate potential has been studied extensively [1, 2, 3]. One of its interesting properties is that there is a Bogomolnytype bound on the energy 进, which is saturated by the self-dual solitons which carry the fractional spin and satisfy the fractional statistics. The potential of this model has two degenerate vacua, implying the existence of topological domain walls interpolating between them. It turns out that the topological domain walls satisfy another Bogomolny bound as a two dimensional model, which is different from such a bound on three dimensional dimensional self-dual solitons [2].

These domain walls which interpolate between the symmetric and asymmetric phases have been studied to understand the behavior of the rotationally symmetric solitons in large charge limit. In this case the energy density is concentrated on a circular ring of large radius, and its radial cross section resembles the domain wall [2]. However in the symmetric phase there exist also rotationally symmetric nontopological solitons with vortices in the center, whose large charge and vorticity limit cannot be described exactly by the topological domain walls. This suggests us a richer structure of domain walls.

On the other hand, recently some two-dimensional non-linear sigma models with appropriate potentials [5] have been shown to have many properties similar to the 4-dimensional Yang-Mills Higgs systems which admit the BPS dyons solutions. In particular, a Noether charge and a topological charge are found to set the BPS-type bound on the energy [ [ 6 , 6 . Since these two-dimensional self-dual models have the underlying $N=2$ supersymmetry and so is our Chern-Simons-Higgs systems, our domain walls are also expected to have the BPS bound, which is more general than the Bogomolny bound.

The goal of this paper is to explore the domain wall structure of the self-dual Chern-SimonsHiggs system, by taking the dimensional reduction of the model to two dimensions. We show that the resultant two dimensional model has a BPS-like energy bound. Our model hence forms a new class of two dimensional models with the BPS-like energy bound, similar to the four dimensional Yang-Mills Higgs systems. We show that the domain wall configurations saturating the energy bound consist of topological and nontopological domain walls: topological domain walls interpolating the symmetric and asymmetric phases, and nontopological domain walls residing in 
the symmetric phase.

The plan of this paper is as follows: In Sec. 2, we introduce our model and find the BPStype energy bound. We reduce the self-dual equations to a single ordinary nonlinear differential equation. In Sec. 3, we solve the differential equation to find and investigate all possible domain wall solutions. In Sec. 4, we study the underlying $N=2$ supersymmetry. In Sec. 5, we study the nonrelativistic limit and its solitons in the symmetric phase. In Sec. 6, we conclude with some remarks. In the appendix, we study another bound on the energy functional, which works only for the topological domain walls, and discuss its relation to the BPS bound.

\section{Model}

We start with the self-dual Abelian Chern-Simons-Higgs system whose Lagrangian [1] is

$$
\mathcal{L}_{C S H}=\frac{\kappa}{2} \epsilon^{\mu \nu \rho} A_{\mu} \partial_{\nu} A_{\rho}+\left|D_{\mu} \phi\right|^{2}-\frac{1}{\kappa^{2}}|\phi|^{2}\left(|\phi|^{2}-v^{2}\right)^{2}
$$

where $D_{\mu} \phi=\left(\partial_{\mu}-i A_{\mu}\right) \phi$. There are two phases in the theory: the symmetric phase where $\phi=0$ and the antisymmetric phase where $\phi=v$. Elementary charged excitations in the symmetric phase has mass $\mu=v^{2} / \kappa$ and spin $s=4 \pi / \kappa$, and in the broken phase there are charge neutral particles of mass $2 \mu$. There are also self-dual anyonic solitons in the symmetric and asymmetric phases.

We first compactify the $y$ direction on a circle of radius $r$ and require that all the fields to be periodic under $y \rightarrow y+2 \pi r$. There are topologically distinct large gauge transformations $e^{i n y / r}$ with any integer $n$. When we expand $A_{y}(t, x, y)$ in Fourier series of $y$, the $y$-independent component $N(t, x)$ becomes periodic due to the large gauge transformations and lies in the interval $[-1 / 2 r, 1 / 2 r]$. In the limit $r=0$, the low energy physics will be dominated by the modes which are independent of the $y$ coordinate.

After we rename $\sqrt{2 \pi r} \phi$ by $\phi, \sqrt{2 \pi r} v$ by $v$, and $2 \pi r \kappa$ by $\kappa$ and keep only the $y$-independent modes, we get the dimensionally reduced Lagrangian in 2 dimensional spacetime,

$$
\mathcal{L}=\kappa N F_{01}+\left|D_{\mu} \phi\right|^{2}-U(N, \phi)
$$

where the potential $U(N, \phi)$ is

$$
U(\phi, N)=N^{2}|\phi|^{2}+\frac{1}{\kappa^{2}}|\phi|^{2}\left(|\phi|^{2}-v^{2}\right)^{2} .
$$


The mass dimension of the fields and parameters become as follows: $[\phi]=[v]=M^{0},\left[A_{\mu}\right]=[N]=$ $M$ and $[\kappa]=M^{-1}$. The kinetic part of $N, A_{\mu}$ is that of the so-called $B F$ theory. To the original Lagrangian, one can add an $\theta$ term,

$$
\mathcal{L}_{\theta}=\theta F_{01},
$$

which is possible only in two dimensions. It does not play any role classically and will be neglected in this paper.

The classical vacuum structure of this theory is determined by the zeroes of the potential $U(\phi, N)$. The symmetric phase $\langle\phi\rangle=0$ is infinitely degenerate as $\langle N\rangle$ can take any value $N_{0}$. In the symmetric phase there are charged scalar bosons of mass

$$
m=\sqrt{N_{0}^{2}+\mu^{2}}
$$

with $\mu=v^{2} / \kappa$ being the three dimensional mass. The asymmetric phase is uniquely given as $<N>=0,<\phi>=v$ up to the gauge transformation. In the asymmetric phase, there are two kinds of neutral bosons of mass $2 \mu$. Since all the vacua are degenerate, we expect there exist topological domain walls. (We call the domain walls residing in the symmetric phase to be nontopological, even though they are topological in a way as they interpolate between different $N$ vacua.)

The Gauss law constraint from the variation of $A_{0}$ is

$$
\kappa N^{\prime}-i\left(D_{0} \phi^{*} \phi-\phi^{*} D_{0} \phi\right)=0
$$

where the prime is the derivative $d / d x$. The variation of $N$ yields another constraint,

$$
\kappa F_{01}-2 N|\phi|^{2}=0
$$

which does not play any important role here.

The theory is invariant under the local gauge transformations

$$
\delta \phi=i \Lambda(x) \phi, \quad \delta A_{\mu}=\partial_{\mu} \Lambda
$$

Its global part is the $U(1)$ symmetry, whose conserved current is $j_{\mu}=i\left(D_{\mu} \phi^{*} \phi-\phi^{*} D_{\mu} \phi\right)$. Making use of the the Gauss law, we can write the corresponding charge as

$$
Q=\int d x j_{0}=\kappa \Delta N
$$


where we introduce $N_{ \pm}=N(t, \pm \infty)$, the constant asymptotic values, and define their difference as $\Delta N=\left(N_{+}-N_{-}\right) . Q / \kappa$ can be identified by the magnetic flux per unit length in three dimensions. There is also a conserved charge, corresponding to the translation invariance along the $y$ axis before the dimensional reduction,

$$
\begin{aligned}
P_{y} & =\int d x i\left(D_{0} \phi^{*} \phi-\phi^{*} D_{0} \phi\right) N \\
& =\frac{\kappa}{2}\left(N_{+}^{2}-N_{-}^{2}\right)=Q \bar{N}
\end{aligned}
$$

where we used the Gauss law and the average of $N$ is defined as

$$
\bar{N}=\left(N_{+}+N_{-}\right) / 2
$$

Thus the $\bar{N}$ can be regarded as the momentum carried by the unit charge along the $y$ direction. If we identify $\bar{N}=N_{0}$, the mass $m=\sqrt{N_{0}^{2}+\mu^{2}}$ of elementary charged particles in the symmetric phase can be thought to be a Lorentz-boosted one from three dimensional mass $\mu$ by the $y$ momentum $N_{0}$.

For the conserved $P_{y}$, there exits the corresponding transformation of the fields,

$$
\delta N=0, \delta \phi=i N \phi, \delta A_{1}=j_{0} / \kappa, \delta A_{0}=j_{1} / \kappa .
$$

This transformation is somewhat puzzling as it does not leave the Lagrangian invariant without the gauge field equation, although the Hamiltonian is invariant modulo the Gauss law. Even though the $y$ space has disappeared, there remains this $y$ directional translation.

Finding the BPS energy bound begins with the explicit expression of the energy density,

$$
\mathcal{E}=\left|D_{0} \phi\right|^{2}+\left|D_{1} \phi\right|^{2}+N^{2}|\phi|^{2}+\frac{1}{\kappa^{2}}|\phi|^{2}\left(|\phi|^{2}-v^{2}\right)^{2}
$$

Together with the Gauss law, one can put the energy density as

$$
\begin{aligned}
\mathcal{E}=\quad & \left|D_{0} \phi+\frac{i}{\kappa} \phi\left(|\phi|^{2}-v^{2}\right) \sin \alpha-i N \phi \cos \alpha\right|^{2} \\
& +\left|D_{1} \phi+\frac{1}{\kappa} \phi\left(|\phi|^{2}-v^{2}\right) \cos \alpha+N \phi \sin \alpha\right|^{2} \\
& +\frac{1}{2 \kappa}\left[\kappa^{2} N^{2}-\left(|\phi|^{2}-v^{2}\right)^{2}\right]^{\prime} \cos \alpha+\left[N\left(v^{2}-|\phi|^{2}\right)\right]^{\prime} \sin \alpha .
\end{aligned}
$$

where $\alpha$ is an arbitrary angle variable. We introduce two charges,

$$
\begin{aligned}
& Y=\int d x \frac{1}{2 \kappa}\left[\kappa^{2} N^{2}-\left(|\phi|^{2}-v^{2}\right)^{2}\right]^{\prime}, \\
& Z=\int d x\left[N\left(v^{2}-|\phi|^{2}\right)\right]^{\prime} .
\end{aligned}
$$


The boundary conditions at spatial infinities for any finite configuration should approach the ground configuration of the potential, $\left(F_{ \pm}=1, N_{ \pm}=0\right)$ or $\left(F_{ \pm}=0, N_{ \pm}=\right.$any value), where $|\phi|^{2}=v^{2} F$. We can rewrite the above two charges as

$$
\begin{aligned}
Y & =P_{y}-\frac{1}{2} \kappa \mu^{2}\left(F_{+}-F_{-}\right)\left(F_{+}+F_{-}-2\right), \\
Z & =\mu Q .
\end{aligned}
$$

Thus we can identify $Y$ as the topological charge and $Z$ as the Noether charge in a broad sense.

From Eq. (14), we can now put a bound on the energy for configurations of a given $Y$ and $Z$ as

$$
E \geq Y \cos \alpha+Z \sin \alpha
$$

for any $\alpha$. It follows that

$$
E \geq \sqrt{Y^{2}+Z^{2}}
$$

This is the BPS-like bound. The bound is saturated by the field configurations satisfying the constraint equations (6) and (7), and the following self-dual equations:

$$
\begin{aligned}
& D_{0} \phi+\frac{i}{\kappa} \phi\left(|\phi|^{2}-v^{2}\right) \sin \alpha-i N \phi \cos \alpha=0, \\
& D_{1} \phi+\frac{1}{\kappa} \phi\left(|\phi|^{2}-v^{2}\right) \cos \alpha+N \phi \sin \alpha=0 .
\end{aligned}
$$

Here $\cos \alpha=Y / \sqrt{Y^{2}+Z^{2}}$ and $\sin \alpha=Z / \sqrt{Y^{2}+Z^{2}}$. The self-dual configurations are static in time as one can show easily that $\partial_{0}|\phi|^{2}=\partial_{0} N=\partial_{x} \operatorname{Arg} \phi-A_{x}=0$. Furthermore, the self-dual configurations also satisfy the usual second-order field equations. The additional constraint (7) is automatically satisfied by the field configurations satisfying the self-dual equations and the Gauss law.

The dimensional reduction of the Bogomolny-type energy bound in three dimensions is identical to the above case with $\sin \alpha= \pm 1$ and so is not a new bound. However in the appendix we will describe another Bogomolny-type bound which works only for the topological domain walls.

Again with $F=|\phi|^{2} / v^{2}$, we can combine the Gauss law (6), and Eqs. (21) and (22) as

$$
\begin{aligned}
& N^{\prime}=-2 \mu F\{\mu(F-1) \sin \alpha-N \cos \alpha\} \\
& F^{\prime}=-2 F\{\mu(F-1) \cos \alpha+N \sin \alpha\}
\end{aligned}
$$


where the prime is $d / d x$. The above equations can be put together as a single ordinary differential equation,

$$
\begin{aligned}
(\ln F)^{\prime \prime} & =-2\left(\mu F^{\prime} \cos \alpha+N^{\prime} \sin \alpha\right) \\
& =-4 \mu^{2} F(1-F) .
\end{aligned}
$$

After integrating this equation once, we obtain

$$
F^{2}-4 \mu^{2} F^{2}\left(F^{2}-2 F+a^{2}\right)=0
$$

where $a$ is an integration constant that lies in $[0,1]$ for any finite energy density solution. For a given $F$ configuration Eq. (24) determines $N$.

There is one different feature in our model compared with the previously studied BPS-type solitons: The length scale of the above equation (26) is independent of the parameter $\alpha$, and so is that of the self-dual domain walls.

\section{Self-dual Solitons}

\section{1 between the symmetric and asymmetric phases}

For $a=1$, the solutions of Eq.(26) are

$$
F=\frac{1}{1+e^{\mp 2 \mu x}}
$$

satisfying $F^{\prime}= \pm 2 \mu F(1-F)$. These solutions describe topological domain walls interpolating between the symmetric and asymmetric phases. The transition region from the symmetric phase to the asymmetric one has the size of order $1 / \mu$. The scalar field $N$ from Eq. (24) is

$$
N=\frac{\mu(\mp 1+\cos \alpha)}{\sin \alpha\left(1+e^{ \pm 2 \mu x}\right)}
$$

Of course the position of the solitons can be translation in space by replacing $x$ by $x-c$. This is the only zero mode of the solution.

The solutions can be classified into two classes depending whether $F_{+}$is one or zero. The first case with $\left(F_{+}, F_{-}\right)=(1,0)$, we get $N_{+}=0$ and $N_{-}=\mu(-1+\cos \alpha) / \sin \alpha$, which fixes $\alpha$ in terms of $N_{-}$. The total energy is

$$
E=\frac{\kappa \mu^{2}(1-\cos \alpha)}{\sin ^{2} \alpha}
$$


The topological charges for this soliton are $Y=E \cos \alpha$ and $Z=E \sin \alpha$. In addition, $Q=-\kappa N_{-}$ and $P_{y}=-\kappa N_{-}^{2} / 2$. Near $\alpha \approx 0$, the wall does not carry any charge and the energy takes $E=\kappa \mu^{2} / 2$, the minimum value of Eq. (29).

The second case is with $\left(F_{+}, F_{-}\right)=(0,1)$, which is the spatial reflection of the first solution. In this case $N_{+}=\mu(1+\cos \alpha) / \sin \alpha$, which fixes the $\alpha$ in terms of $N_{+}$, and $N_{-}=0$. The energy of the soliton is

$$
E=\frac{\kappa \mu^{2}(1+\cos \alpha)}{\sin ^{2} \alpha}
$$

Again the charges are uniquely determined by $N_{+}$or the angle $\alpha$.

\subsection{In the symmetric phase}

For $a<1$, the general solution of Eq. (26) is given by

$$
F=\frac{a^{2}}{1+\sqrt{1-a^{2}} \cosh (2 a \mu x)} .
$$

Since $F_{ \pm}=0$, this domain wall lives in the symmetric phase. From Eq.(24), we get

$$
N=\left(\frac{\mu \sqrt{1-a^{2}}}{\sin \alpha}\right)\left(\frac{a \sinh (2 a \mu x)+\left[\sqrt{1-a^{2}}+\cosh (2 a \mu x)\right] \cos \alpha}{1+\sqrt{1-a^{2}} \cosh (2 a \mu x)}\right)
$$

where $N_{+}=\mu(a+\cos \alpha) / \sin \alpha$ and $N_{-}=-\mu(a-\cos \alpha) / \sin \alpha$. The energy is given by

$$
E=\frac{2 a \kappa \mu^{2}}{\sin ^{2} \alpha}
$$

Thus, for given $\alpha$ and $a$, there is a unique solution up to the translation. Again, $Y$ and $Z$ are $E \cos \alpha$ and $E \sin \alpha$.

Let us reconsider the above configuration somewhat differently. The average of $N$ is $\bar{N}=\mu \cot \alpha$. This fixes the angle $\alpha$. The difference of $N$ is given by $\Delta N=2 \mu a / \sin \alpha$, which fixes $a$ in terms of $\bar{N}$ and $\Delta N$. Since the electric charge is $Q=\kappa \Delta N$, the energy (33) can be expressed as

$$
E=Q \sqrt{\bar{N}^{2}+\mu^{2}}
$$

Since the mass of elementary charged particles in the symmetric phase is $m=\sqrt{N_{0}^{2}+\mu^{2}}$, this soliton can be regarded as a Q-ball lump of elementary particles if we identify the vacuum value $N_{0}$ by the average $\bar{N}$. 
In the limit $a \rightarrow 1$, the size of this soliton gets very large. The spatial dependence is characterized by the parameter $\mu$. As $x$ increases, the value of $F$ jumps from zero to a value approximately one in a wall of size $1 / \mu$, then stays there for the interval of approximate size $\left(-\ln \sqrt{1-a^{2}}\right) / 2 \mu$, then falls down to zero in the wall of size $1 / \mu$. From the spatial dependence of $F$ and $N$, one can see that in this limit the soliton looks more and more like a combination of two topological solitons considered in the previous subsection.

Especially when we choose $\bar{N}=0$ or $\cos \alpha=0$, the limit $Q=2 \kappa \mu a \rightarrow 2 v^{2}$ as $a \rightarrow 1$. If the semiclassical picture should be correct, the charge of this soliton should be much larger than that of elementary particles, or $2 v^{2} \gg 1$.

\section{Supersymmetry}

In Ref. [7] the underlying $N=2$ supersymmetry theory for the self-dual Chern-Simons Higgs systems has been found. After the dimensional reduction of this model, we get again an $N=2$ supersymmetric model. Here we use the convention that $\eta_{\mu \nu}=\operatorname{diag}(1,-1,-1)$, and $\gamma^{0}=\sigma_{2}, \gamma^{1}=$ $i \sigma_{1}, \gamma^{2}=-i \sigma_{3}$. After the dimensional reduction, we introduce $\gamma^{5}=\gamma_{5} \equiv \gamma^{0} \gamma^{1}=\sigma_{3}$. The supersymmetric Lagrangian in two dimensions becomes

$$
\begin{aligned}
\mathcal{L}_{\text {susy }}= & \kappa N F_{01}+\left|D_{\mu} \phi\right|^{2}-N^{2}|\phi|^{2}-\frac{1}{\kappa^{2}}|\phi|^{2}\left(|\phi|^{2}-v^{2}\right)^{2} \\
& +i \bar{\psi} \gamma^{\mu} D_{\mu} \psi-i N \bar{\psi} \gamma^{5} \psi+\frac{1}{\kappa}\left(3|\phi|^{2}-v^{2}\right) \bar{\psi} \psi
\end{aligned}
$$

After the dimensional reduction, the supersymmetric transformation becomes

$$
\begin{aligned}
& \delta A_{\mu}=\frac{1}{\kappa}\left(\bar{\zeta} \gamma_{\mu} \psi \phi^{*}+\bar{\psi} \gamma_{\mu} \zeta \phi\right), \\
& \delta N=\frac{i}{\kappa}\left(\bar{\zeta} \gamma^{5} \psi \phi^{*}+\bar{\psi} \gamma^{5} \zeta \phi\right), \\
& \delta \phi=\bar{\zeta} \psi, \\
& \delta \psi=-i \gamma^{\mu} \zeta D_{\mu} \phi+i \gamma^{5} \zeta N \phi+\frac{1}{\kappa} \phi\left(|\phi|^{2}-v^{2}\right) \zeta .
\end{aligned}
$$

Since the parameter $\zeta$ is a complex Dirac spinor, it generates the $N=2$ supersymmetry transformation. (We can choose the chiral spinors $\left(1 \pm \gamma^{5}\right) \zeta / 2$ as independent parameters and so the supersymmetry is really $N=(2,2)$.) 
For a given superfield $\Phi$ the supersymmetric transformation

$$
\delta \Phi=[i(\bar{\zeta} \mathcal{R}+\overline{\mathcal{R}} \zeta), \Phi]
$$

is generated by the supercharge

$$
\mathcal{R}=\int d x\left\{\left[\left(D_{\mu} \phi\right)^{*} \gamma^{\mu}+N \phi^{*} \gamma^{5}-\frac{i}{\kappa} \phi^{*}\left(|\phi|^{2}-v^{2}\right)\right] \gamma^{0} \psi\right\}
$$

After quantization, we get the canonical commutation relations between the fields. These lead to the relevant $N=2$ superalgebra,

$$
[\bar{\zeta} \mathcal{R}, \overline{\mathcal{R}} \eta]=\bar{\zeta} \gamma^{\mu} \eta P_{\mu}-\bar{\zeta} \eta Z+i \bar{\zeta} \gamma^{5} \eta Y
$$

where two central charges are given as

$$
\begin{aligned}
& Y=\int d x\left\{N\left[i\left(D_{0} \phi^{*} \phi-\phi^{*} D_{0} \phi\right)-\psi^{\dagger} \psi\right]-\frac{1}{2 \kappa} \partial_{x}\left(|\phi|^{2}-v^{2}\right)^{2}\right\} \\
& \left.Z=-\int d x\left\{N \partial_{x}|\phi|^{2}+\frac{1}{\kappa}\left(|\phi|^{2}-v^{2}\right)\left[i\left(D_{0} \phi^{*} \phi-\phi^{*} D_{0} \phi\right)-\psi^{\dagger} \psi\right)\right]\right\} .
\end{aligned}
$$

These central charges become those in Eqs. (15) and (16) once the Gauss law (6) is used.

After introducing a new spinor operator,

$$
\begin{aligned}
\mathcal{R}^{\prime}=\frac{1}{2}\left(1+\gamma_{0} e^{i(\pi / 2-\alpha) \gamma_{5}}\right) \mathcal{R} & \\
=\frac{1}{2}\left(1+\gamma_{0} e^{i(\pi / 2-\alpha) \gamma_{5}}\right) \int d x\{\psi & {\left[\left(D_{0} \phi\right)^{*}-\frac{i}{\kappa} \phi^{*}\left(|\phi|^{2}-v^{2}\right) \sin \alpha+i N \phi^{*} \cos \alpha\right] } \\
-\gamma_{5} \psi & {\left.\left[\left(D_{1} \phi\right)^{*}+\frac{1}{\kappa} \phi^{*}\left(|\phi|^{2}-v^{2}\right) \cos \alpha+N \phi^{*} \sin \alpha\right]\right\}, }
\end{aligned}
$$

the superalgebra (39) becomes

$$
\sum_{\beta}\left\{\mathcal{R}_{\beta}^{\prime}, \mathcal{R}_{\beta}^{\prime \dagger}\right\}=E-(Y \cos \alpha+Z \sin \alpha)
$$

As the left-handed side of this equation is positive definite, this equation leads to the energy bound (19). The energy bound is saturated when $\left\langle\mathcal{R}^{\prime}\right\rangle=0$, which in turn implies the self-dual equations.

\section{The Nonrelativistic Limit}

We expect the nonrelativistic limit of the theory can be taken in the symmetric phase as the field $\phi$ describes charged particles. We expect the nonrelativistic limit is reasonable if the kinetic energy 
is small. For this limit to make a sense, it turns out that the $y$ momentum per unit charge should be also small compare with the three-dimensional mass, or $\left|N_{0}\right| \ll \mu$.

In this case we take the non-relativistic limit of the system in the three dimensional model by letting $\phi=(1 / \sqrt{2 \mu}) e^{-i \mu t} \psi$, where $\mu=v^{2} / \kappa$. The Lagrangian (1) reduces to the well known Lagrangian [8],

$$
\mathcal{L}_{\text {nonrel }}=\frac{\kappa}{2} \epsilon^{\mu \nu \rho} A_{\mu} \partial_{\nu} A_{\rho}+i \psi^{*} D_{t} \psi-\frac{1}{2 \mu}\left|D_{\mu} \psi\right|^{2}+\frac{1}{2 \mu \kappa}\left(|\psi|^{2}\right)^{2} .
$$

After dimensional reduction, it becomes

$$
\mathcal{L}_{\text {nonrel }}=\kappa N F_{01}+i \psi^{*} D_{t} \psi-\frac{1}{2 \mu}\left|D_{x} \psi\right|^{2}-\frac{1}{2 \mu} N^{2}|\psi|^{2}+\frac{1}{2 \mu \kappa}\left(|\psi|^{2}\right)^{2} .
$$

This is equivalent to the nonrelativistic limit of the dimensionally reduced Lagrangian if $N_{0} \ll \mu$. The energy functional, after dropping the boundary term which depends only on the conserved charge, is given by

$$
E_{\text {nonrel }}=\int d x\left\{\frac{1}{2 \mu}\left|D_{x} \psi\right|^{2}+\frac{1}{2 \mu} N^{2}|\psi|^{2}-\frac{1}{2 \mu \kappa}\left(|\psi|^{2}\right)^{2}\right\} .
$$

This energy is reasonable as its density is gauge invariant. We split the $N$ field as a sum of the average $\bar{N}$ and the fluctuation $\delta N$ such that $\delta N_{+}+\delta N_{-}=0$.

By making use of the Gauss law $\kappa N^{\prime}+|\psi|^{2}=0$, the energy functional can be rewritten as

$$
E_{\text {nonrel }}=\frac{1}{2 \mu} \bar{N}^{2}|Q|+\int d x\left\{\frac{1}{2 \mu}\left|D_{x} \psi-\delta N \psi\right|^{2}\right\}
$$

plus the vanishing boundary terms, where $Q=-\int d x|\psi|^{2}<0$. Since the integrand is nonnegative, the nonrelativistic energy is bounded by the $y$ directional kinetic energy, $\bar{N}^{2}|Q| /(2 \mu)$, which can be seen as the mass correction of the $Q$ particles by $P_{y}=\bar{N}$.

Introducing $|\psi|^{2}=2 \mu v^{2} F$, we can simplify the Gauss law and the nonrelativistic self-dual equations to

$$
\begin{aligned}
& \delta N^{\prime}+2 \mu^{2} F=0, \\
& F^{\prime}-2 \delta N F=0 .
\end{aligned}
$$

They in turn lead to the following equations

$$
\begin{array}{r}
(\ln F)^{\prime \prime}+4 \mu^{2} F=0, \\
\delta N=(\ln F)^{\prime} / 2 .
\end{array}
$$


The equation (50) is the Liouville equation in one dimension, which can be integrated to

$$
F^{2}+4 \mu^{2} F^{2}\left(2 F-a^{2}\right)=0
$$

where $0<a<1$. This is again the nonrelativistic limit of Eq. (26) with the same parameter $a$. The solutions are given by

$$
\begin{aligned}
& F=\frac{a^{2}}{2 \cosh ^{2}(a \mu x)}, \\
& N=N_{0}-\frac{a \mu}{2} \tanh (a \mu x) .
\end{aligned}
$$

In the large $x$ region the above nonrelativistic solutions match with the relativistic solution (31) if $a \ll 1, \sin \alpha=-1$. The charge of this configuration is again $Q=2 \kappa \mu a$.

\section{Concluding Remarks}

Here we have explored the structure of the domain walls in the self-dual Chern-Simons-Higgs systems by the dimensional reduction method. Their energy bound is similar to the BPS bound on dyons in some Yang-Mills Higgs systems in four dimensions. We found all possible domain solutions, which are made of topological and nontopological domain walls. We studied the $N=2$ supersymmetry behind the BPS-like energy bound. We also have studied the nonrelativistic limit.

Our two dimensional model is different from the recent attempts to describe anyons in one dimensional space [9]. Neither our model seems to be directly related to the Calogero-Suthutherland model, contrast to the recent work [10]. Our nonrelativistic limit is simpler than the recently discovered chiral solitons [11]. However, it turns out that our nonrelativistic model has also intriguing properties as shown in a recent work [12].

There are several directions to explore from this point. There are self-dual Chern-Simons-Higgs models for any gauge group and any matter representation [13, 14]. Especially with the matter in the adjoint representation, the vacuum structure is quite rich [15, 16], implying the intricate domain wall structure. As in the abelian case, domain walls in two dimensions are generally easier to understand than solitons in three dimensions, and such understanding will eventually lead to a better understanding of solitons in three dimensions.

We studied the $N=2$ supersymmetry underlying the self-duality. However, in three dimensions with the Chern-Simons term the maximal supersymmetry is $N=3$ [18], which would be translated 
to the $N=3$ supersymmetric theory in two dimensions. As a pure two dimensional system, it is interesting to find out how the $N=3$ supersymmetry can be maximal. One may wonder whether there exist a more general set of the self-dual systems with the $B F$ kinetic termin two dimensions with larger supersymmetry than the $N=3$ supersymmetry.

Along the similar line of thought, there may be a gauged version of the nonlinear sigma models considered in Ref. [5]. They would lead to a richer variety of two dimensional models with the BPS-type energy bound, which may be similar to the dimensional reduction of the self-dual $C P(N)$ models considered in three dimensions [17].

The quantum mechanical aspect of the theory should be also interesting. In the massless limit $v=0$, there may be a quantum conformal symmetry. Also the three dimensional solitons may appear as instantons in two dimensions, whose effect is unclear at this moment. One of the noticeable feature here is that the Euclidean action is complex and so the instanton solutions should be treated carefully as in the case of monopole instantons in three dimensional Chern-Simons-Higgs systems [19].

\section{Acknowledgements}

The work of H.-C. K. was supported by the National Science Council of Taiwan-Republic of China under the contract number No. NSC 86-2112-M-032-011-T. The work of K.L. was supported by the US Department of Energy and the NSF Presidential Young Investigator Fellowship. The work of T.L. was supported by the Basic Research Institute Program, Ministry of Education, Project BSRI-96-2401 and in part by KOSEP through the Center for Theoretical Physics of Seoul National University. 


\section{Appendix: Another bound}

In this appendix, we briefly review another Bogomolny-type bound which works only for the topological domain walls [2]. We can rewrite the energy (13) as follows:

$$
\begin{array}{cl}
\mathcal{E}=\quad & \left|D_{0} \phi \pm i N \phi\right|^{2}+\left|D_{1} \phi \pm \frac{1}{\kappa} \phi\left(|\phi|^{2}-v^{2}\right)\right|^{2} \\
& \mp \frac{1}{2 \kappa}\left[\kappa^{2} N^{2}+\left(|\phi|^{2}-v^{2}\right)^{2}\right]^{\prime} .
\end{array}
$$

This is not identical to the equation (14) with $\cos \alpha= \pm 1$ because of the sign difference in the boundary term. The energy bound is then

$$
E \geq\left|\int d x \frac{1}{2 \kappa}\left[\kappa^{2} N^{2}+\left(|\phi|^{2}-v^{2}\right)^{2}\right]^{\prime}\right|
$$

Let us concentrate on the upper sign. The self-dual equations in this case become

$$
\begin{aligned}
& \kappa N^{\prime}=-2|\phi|^{2} \\
& D_{1} \phi+\frac{1}{\kappa} \phi\left(|\phi|^{2}-v^{2}\right)=0
\end{aligned}
$$

where we have used the Gauss law (6). The $\phi$ equation leads to the topological domain wall solution (27) with $\left(F_{+}, F_{-}\right)=(1,0)$. The $N$ equation has a nontrivial solution (28) with $\left(N_{+}=0, N_{-}>0\right)$.

Seemingly different two sets of self-dual equations are satisfied by the fields for the same topological domain wall. The new energy bound (56) is identical to the BPS energy (29) for these configurations. We believe that the presence of this additional energy bound is due to the interdependence of two charges $Y$ and $Z$ for the topological domain walls. While the above bound does not lead to anything new in the abelian self-dual case, its analogue in the nonabelian case may be more useful. 


\section{References}

[1] J. Hong, Y. Kim and P-Y. Pac, Phys. Rev. Lett. 64, 2330 (1990); R. Jackiw and E. Weinberg, Phys. Rev. Lett. 64, 2334 (1990).

[2] R. Jackiw, K. Lee and E.J. Weinberg, Phys. Rev. D42, 3488 (1990).

[3] Y. Kim and K. Lee, Phys. Rev. D49, 2041 (1994).

[4] E. Bogomolny, Sov. J. Nucl. Phys. 24, 449 (1976).

[5] E.R.C. Abrahm and P.K. Townsend, Nucl. Phys. B 351, 313 (1991); Phys. Lett. B291, 85 (1992); Phys. Lett. B295, 225 (1992); A. Opfermann and G. Papadopoulos, Solitons in (1,1)supersymmetric massive sigma models, hep-th/9610099.

[6] M.K. Prasad and C.M. Sommerfield, Phys. Rev. Lett. 35, 760 (1975); S. Coleman, S. Parke, A. Neveu and C.M. Sommerfield, Phys. Rev. D15, 544 (1977).

[7] C. Lee, K. Lee and E. Weinberg, Phys. Lett. B 243, 105 (1990).

[8] R. Jackiw and S.-P. Pi, Phys. Rev. Lett. 64, 2969 (1990); Phys. Rev. D 42, 3500 (1990).

[9] S.J. Rabello, Phys. Lett. B363, 180 (1995)

[10] I. Andrić, V. Bardek and L. Jonke, Calogero-Sutherland model from excitations of ChernSimons vortices, hep-th/9507110.

[11] U. Aglietti, L. Griguolo, R. Jackiw, S.-Y. Pi, and D. Seminara, Phys. Rev. Lett. 77, 4406 (1996); R. Jackiw, A Nonrelativistic Chiral Soliton in One Dimension, hep-th/9611185.

[12] P. Oh and C. Rim, Solitons in the Chern-Simons Inspired $1+1$ D Field Theory, hepth/9612028.

[13] K. Lee, Phys. Lett. B255, 381 (1991); K. Lee, Phys. Rev. Lett. 66, 553 (1990).

[14] G. Dunne, Phys. Lett. B324, 359 (1994).

[15] H-C. Kao and K. Lee, Phys. Rev. D50, 6626 (1994). 
[16] G. Dunne, Nucl. Phys. B433, 333 (1995); G. Dunne, Phys. Lett. B345, 452 (1995).

[17] K. Kimm, K. Lee and T. Lee, Phys. Rev. D53, 4436 (1995).

[18] H.-C. Kao and K. Lee, Phys. Rev. D46, 4691 (1992); H.-C. Kao, Phys. Rev. D50, 2881 (1994).

[19] K. Lee, Nucl. Phys. B373, 735 (1992). 